\title{
Modeling of influence of stresses on indicators of resistance of mining rocks to mechanical destruction
}

\author{
Serhii Tynyna, ${ }^{1, *}$, Ihor Chobotko ${ }^{2}$, Liliya Frolova $^{3}$, and Tetyana Butyrina ${ }^{3}$ \\ ${ }^{1}$ Institute of Geotechnical Mechanics named by N. Poljakov of National Academy of Sciences of \\ Ukraine, 49005, Dnipro, Simferopolska Str., 2a, Ukraine \\ ${ }^{2}$ National Technical University Dnipro Polytechnic, 49000, Dnipro, Dmytra Yavornytskoho Ave., 19, \\ Ukraine \\ ${ }^{3}$ Ukrainian State University of Chemical Technology, 49005, Dnipro, Haharina Ave., 8, Ukraine
}

\begin{abstract}
The article deals with the problem of determining the expression for the components of the stress tensor of a rock mass that is exposed to rock-breaking tools. In solving this problem, the natural tension of the rocks and the stresses arising ahead, in the plane and on the boundary of the face of the cylindrical excavation were taken into account. Formulation presented in the form of a semi-infinite cylinder whose axis coincides with the axis $\bar{z}$ of the coordinate system. The solution is obtained in a cylindrical coordinate system in the form of analytical expressions, which is carried out by numerical integration of improper integrals.
\end{abstract}

\section{Introduction}

In determining the effectiveness of mechanical rock-breaking tools, contact strength is taken as the main indicator of the resistance of rocks to destruction $P_{K}$.

A significant part of the recently developed rocks under the influence of rock-cutting tool has a brittle fracture. It is characterized by the fact that, at a certain amount of contact stresses, a cone-shaped hole is formed at the boundary of the seal core. Therefore, the correlation between the contact resistance and tensile strength $P_{K}$ brittle rocks can be used to calculate the contact tensile strength $[1,2]$.

During the excavation of the stress-strain state of a rock mass is very difficult and controversial. Studies [3, 4] have shown that in the vicinity of the face of cylindrical production in its roof there is a zone of tensile radial stresses, while the area of the massif in front of the face is under the influence of significant compressive stresses.

As the distance increases $\bar{z}_{0}\left(\bar{z}_{0} \approx 20 \mathrm{~m}\right)$ from the mining area, a flat stress state is realized in the massif. In the theoretical studies carried out to date on the question of contact strength $[5,6]$, the authors used well-known analytical dependences for the stress tensor components from the Boussinesq decision [7] or from the decision of the contact problem of the theory of elasticity [8].

In these decisions, to the corresponding expressions for the stress tensor components

\footnotetext{
*Corresponding author: haritonroots@gmail.com
} 
were added natural voltage untouched array, and then the stress distribution graphs were constructed on the basis of which conclusions were made on the contact rock strength in a near zone of the array.

It is easy to see that the analytical dependencies for stresses used in this way do not take into account the influence of the perturbed stress field caused by the mine workings. Therefore, it is rather difficult to judge the reliability of the findings obtained in this way about the contact strength of rocks.

It is therefore necessary to develop a technique for solving problems in order to obtain analytical expressions for the stresses taking into account all factors characterizing stressstrain state of the rock mass in the process of production.

The decision of the external problem of the stress-strain state of the rock mass when carrying out horizontal cylindrical workings in it was obtained in [3, 4].

As a result of decision this problem, it has been established that on the surface $r=h$ ( $h$ - the radius of development), limiting the rock mass in front of the face, there is a significant concentration of radial (compressive) $\sigma_{r}$ and tangential $\sigma_{r z}$ stresses.

This character of the stress state in the rock mass significantly affect contact strength rocks and, hence, to working conditions rock cutting tools.

Thus, to determine the contact strength of the rock mass, exposed to the rock-breaking tool, it is necessary to determine the analytical dependencies for the stress tensor components, taking into account the natural stress of the rocks and stresses that occur in the rock mass as a result of technological impact during penetration development.

\section{Problem statement}

Consider the stresses $P_{0}$ that occur in front of the face of a cylindrical excavation in the region representing a semi-infinite cylinder, whose axis coincides with the axis $\bar{z}$ of the coordinate system (Fig. 1).
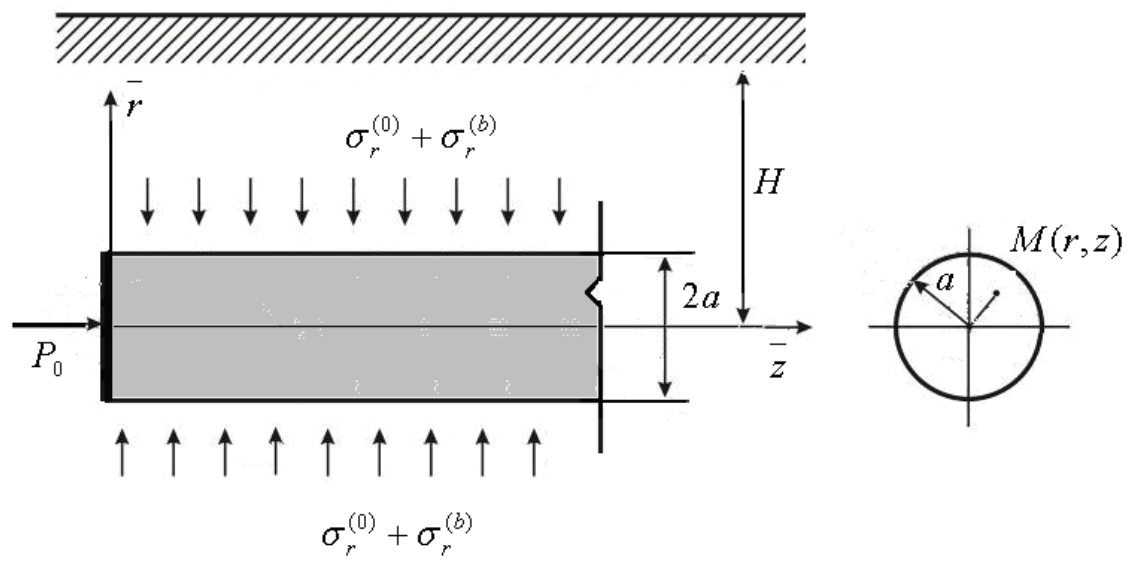

Fig. 1. Scheme to the decision of the problem of the stressed state of the rock massif ahead of slaughtering.

Suppose that the rock mass in this area is deformed as a result of:

- the actions of natural stresses caused by the formation of the developed space during mining [1]:

$$
\sigma_{r r}^{(0)}=-\gamma H ; \sigma_{z z}^{(0)}=-\lambda_{0} \gamma H ; \sigma_{r z}^{(0)}=0 ;
$$


- concentrated effort caused by the influence of rock cutting tool:

$$
\begin{gathered}
\sigma_{r r}^{(b)}=-\bar{z}_{0} \gamma H\left\{\sqrt{\left(\bar{z}+\bar{z}_{0}\right)^{2}-z_{0}^{2}}\left[\bar{z}+\bar{z}_{0}+\sqrt{\left(\bar{z}+\bar{z}_{0}\right)^{2}-z_{0}^{2}}\right]\right\}^{-1} \\
\sigma_{r z}^{(b)}=(1-2 v) \gamma H\left[\pi \bar{z}_{0} \sqrt{\left(\bar{z}+\bar{z}_{0}\right)^{2}-z_{0}^{2}}\right]^{-1}
\end{gathered}
$$

where in (1) and (2) are indicated: $\sigma_{r r}{ }^{(0)}$ and $\sigma_{r r}{ }^{(b)}, \sigma_{z z}{ }^{(0)}$ and $\sigma_{z z}{ }^{(b)}$ - are normal stresses, $\sigma_{r z}{ }^{(b)}$ - are tangential stresses; $H$ - the depth from the earth surface to the axis of generation; $v$ - Poisson's ratio; $\gamma$ - volume weight of the rock mass; $\lambda_{0^{-}}$coefficient pressure ratio; $\overline{z_{0}} \approx 10 a$.

Since the depth of production $H>>2 a$, then the change in natural stresses along the height of production can be neglected. Therefore, in the course of further research, we will assume that the stresses caused by the workings are set on the lateral surface of the semiinfinite cylinder, and the end face of the cylindrical body is subject to the technological impact of the rock-destructive tool.

To meet one of the requirements of a physical model of the physical and mechanical properties of the rocks will be characterize by relations of linearly elastic deformable body.

In view of the foregoing, the boundary conditions of the problem can be represented in this form:

$$
\begin{gathered}
\text { at } r=1, \sigma_{r r}=Q_{r r}(z) ; \quad \sigma_{r z}=Q_{r z}(r) ; 0 \leq z \leq \infty \\
\sigma_{r r}(z)=-\gamma H \bar{z}_{0}\left\{\sqrt{\left(\bar{z}+\bar{z}_{0}\right)^{2}-z_{0}^{2}}\left[\bar{z}+\bar{z}_{0}+\sqrt{\left(\bar{z}+\bar{z}_{0}\right)^{2}-z_{0}^{2}}\right]\right\}^{-1} ; \\
\sigma_{r z}(z)=(1-2 v) \gamma H\left[\pi \bar{z}_{0} \sqrt{\left(\bar{z}+\bar{z}_{0}\right)^{2}-z_{0}^{2}}\right]^{-1} ; \\
\text { at } z=0, \sigma_{r z}=Q_{z z}(r) ; \sigma_{r z}=0 ; Q_{z z}=-P_{0} \exp \left[c\left(r-r_{0}\right)\right]+\lambda_{0} \gamma H .
\end{gathered}
$$

The defining equations of an elastically deformable body can be represented in this form [3]:

$$
\begin{gathered}
\sigma_{\theta_{1} \theta_{1}}=q_{0}\left[\frac{v}{1-v}\left(\frac{\partial U_{r}}{\partial r}+\frac{\partial U_{z}}{\partial z}\right)+\frac{U_{r}}{r}\right] \\
\frac{\partial}{\partial z}\left(\frac{\partial}{\partial r}+\frac{1}{r}\right) U_{r}+(1-2 v)\left[\frac{\partial^{2}}{\partial r^{2}}+\frac{1}{r}+\overline{\beta_{2}^{2}} \frac{\partial^{2}}{\partial z^{2}}\right] U_{z}=0,
\end{gathered}
$$

where $r$ and $z$ - dimensionless coordinates, $r=r a^{-1} ; z=\bar{z} a^{-1} ; a$ - the radius of the cylindrical cavity; $U_{r}$ and $U_{z}$ - components of the displacement vector respectively in directions $\bar{r}$ and $\bar{z} ; E$ - Young's modulus; $\overline{\beta_{1,2}}$ - constants having the form:

$$
\begin{gathered}
\overline{\beta_{2}^{2}}=\frac{2(1-v)}{1-2 v} \cdot \overline{\beta_{1}^{2}}=\frac{1-2 v}{2(1-v)} \\
\sigma_{\theta \theta}=q_{0}\left[\frac{v}{1-v}\left(\frac{\partial U_{r}}{\partial r}+\frac{\partial U_{z}}{\partial z}\right)+\frac{U_{r}}{r}\right] ; \quad \sigma_{r r}=q_{0}\left[\frac{\partial U_{r}}{\partial r}+\frac{v}{1-v}\left(\frac{U_{r}}{r}+\frac{\partial U_{z}}{\partial z}\right)\right] ;
\end{gathered}
$$




$$
\begin{gathered}
\sigma_{z z}=q_{0}\left[\frac{v}{1-v}\left(\frac{\partial U_{r}}{\partial r}+\frac{U_{r}}{r}\right)+\frac{\partial U_{z}}{\partial z}\right] ; \sigma_{r z}=q_{1}\left[\frac{\partial U_{r}}{\partial z}+\frac{\partial U_{z}}{\partial r}\right] ; \\
q_{0}=\frac{1-v}{a(1+v)(1-2 v)} E ; \quad q_{1}=\frac{E}{2 a(1+v)} .
\end{gathered}
$$

It should be noted that, in a closed form, the decision of problems similar to those formulated above was obtained only for a semi-infinite cylinder.

The main difficulty of the tasks of the stress-deformed state of the cylinder of finite dimensions is that by using known decisions of equilibrium equations into a cylindrical coordinate system is not possible directly to satisfy the conditions on the side surface and the ends of the cylinder.

\section{Decision}

Known general decisions of the equations of dynamic equilibrium in a cylindrical coordinate system. In these equations, the components of the vector of elastic displacements are expressed through functions that meet the equilibrium equations $[3,5]$. But they do not provide the necessary functional arbitrariness to meet the boundary conditions on the lateral surface (3) and the ends of the cylindrical body (4).

In this regard, it is necessary to apply approaches to decision equilibrium equations (4) in a form that allows one to strictly meet the boundary conditions on the side surface $\bar{r}=a$ and at the ends $z=0$ cylindrical body.

The decision of the system of equations (5) we will be sought in the form:

$$
U_{r}=\frac{\partial \varphi}{\partial r}-\frac{b-\lambda c}{1-\lambda b} \frac{\partial \psi}{\partial z} ; U_{z}=\left[\frac{a-c}{1-\lambda}+2(1-v) \frac{b}{1-\lambda b}\right]\left(\frac{\partial}{\partial r}+\frac{1}{r}\right) \psi-\frac{\partial \varphi}{\partial z},
$$

where $\varphi(r, z)$ and $\psi(r, z)$ - some functions; $\lambda, a, b, c$ - arbitrary constants.

Substituting expressions (4) into equations (5) and making the corresponding rearrangement of the terms, we obtain the following system of differential equations:

$$
L_{11} \frac{\partial \varphi}{\partial r}+\omega_{1} L_{12} \frac{\partial \psi}{\partial z}=0 ; 2 v L_{21} \frac{\partial \varphi}{\partial z}+\omega_{2} L_{22}\left(\frac{\partial}{\partial r}+\frac{1}{r}\right) \psi=0
$$

where $L_{i j}(i, j=1,2)$ - second order differential operators.

The equations can be represented in this form:

$$
L_{11} \phi_{1}=0 ; L_{12} \phi_{2}=0,
$$

where marked

$$
\phi_{1}=\frac{\partial \varphi}{\partial r}+\omega_{1} \frac{\partial \psi}{\partial z} ; \phi_{2}=2 v \frac{\partial \varphi}{\partial r}+\omega_{2}\left(\frac{\partial}{\partial r}+\frac{1}{r}\right) \psi
$$

The decision of equations (9) is found using the Fourier method [8] for $A_{j j}(\alpha)$ and $B_{j j}(\alpha)$ $(j=1,2)$ - arbitrary function argument, where $\alpha ; i_{0}=\sqrt{-1} ; J_{n}\left(\alpha \beta_{j} r\right)$ and $Y_{n}\left(\alpha \beta_{j} r\right),(n=0,1$; $j=1,2)$ - Bessel functions of $n$ - order of the first and the second kind, respectively.

As a result, we obtain the following system of inhomogeneous differential equations 


$$
\begin{gathered}
\frac{\partial \varphi}{\partial r}+\omega_{1} \frac{\partial \psi}{\partial z}=\frac{1}{2 \pi} \int_{-\infty}^{\infty}\left[A_{11}(\alpha) J_{1}\left(\alpha \beta_{1} r\right)+B_{11}(\alpha) Y_{1}\left(\alpha \beta_{1} r\right)\right] \exp \left(i_{0} \alpha z\right) d \alpha \\
2 v \frac{\partial \varphi}{\partial r}+\omega_{2}\left(\frac{\partial}{\partial r}+\frac{1}{r}\right) \psi=\frac{1}{2 \pi} \int_{-\infty}^{\infty}\left[A_{22}(\alpha) J_{0}\left(\alpha \beta_{2} r\right)+B_{11}(\alpha) Y_{0}\left(\alpha \beta_{2} r\right)\right] \exp \left(i_{0} \alpha z\right) d \alpha .
\end{gathered}
$$

Alternately excluding the functions in (10) $\varphi$ and $\psi$ and taking into account the following properties of the Bessel functions, we arrive at two inhomogeneous differential equations for the desired functions $\varphi(r, z)$ and $\psi(r, z)$ :

$$
\begin{gathered}
{\left[\frac{\partial^{2}}{\partial r^{2}}+\frac{1}{r} \frac{\partial}{\partial r}-2 v \frac{\omega_{1}}{\omega_{2}} \frac{\partial^{2}}{\partial z^{2}}\right] \varphi=\frac{1}{2 \pi} \int_{-\infty}^{\infty}\left\{\alpha \beta_{1} A_{11}(\alpha) J_{0}\left(\alpha \beta_{1} r\right)+\alpha \beta_{1} B_{11}(\alpha) Y_{0}\left(\alpha \beta_{1} r\right)+\right.} \\
\left.+i_{0} \alpha \frac{\omega_{1}}{\omega_{2}}\left[A_{22}(\alpha) J_{0}\left(\alpha \beta_{2} r\right)+B_{22}(\alpha) Y_{0}\left(\alpha \beta_{2} r\right)\right]\right\} \exp \left(i_{0} \alpha z\right) d \alpha \\
{\left[\frac{\partial^{2}}{\partial r^{2}}+\frac{1}{r} \frac{\partial}{\partial r}-\frac{1}{r^{2}}-2 v \frac{\omega_{1}}{\omega_{2}} \frac{\partial^{2}}{\partial z^{2}}\right] \psi=} \\
=-\frac{1}{2 \pi} \int_{-\infty}^{\infty}\left\{\frac{\alpha \beta_{2}}{\omega_{2}}\left[A_{22}(\alpha) J_{1}\left(\alpha \beta_{2} r\right)+B_{22}(\alpha) Y_{1}\left(\alpha \beta_{2} r\right)\right]-\right. \\
\left.-i_{0} \alpha_{0} \frac{2 v}{\omega_{2}}\left[A_{11}(\alpha) J_{1}\left(\alpha \beta_{1} r\right)+B_{11}(\alpha) Y_{1}\left(\alpha \beta_{1} r\right)\right]\right\} \exp \left(i_{0} \alpha z\right) d \alpha .
\end{gathered}
$$

The decision of equations is the sum of

$$
\varphi=\bar{\varphi}+\stackrel{=}{\varphi} ; \psi=\bar{\psi}+\bar{\psi},
$$

where indicated $\bar{\varphi}$ and $\bar{\psi}$ - general decisions of homogeneous ones, but $\overline{\bar{\varphi}}$ and $\overline{\bar{\psi}}$ particular decisions of the corresponding inhomogeneous equations (11).

We find homogeneous decisions by the method of separation of variables, and we write non-homogeneous solutions in the form:

$$
\begin{gathered}
\varphi=\frac{1}{2 \pi} \int_{-\infty}^{\infty}\left\{\frac{\alpha^{-1} \beta_{1}}{\beta_{3}^{2}-\beta_{1}^{2}}\left[A_{11}(\alpha) J_{0}\left(\alpha \beta_{1} r\right)+B_{11}(\alpha) Y_{0}\left(\alpha \beta_{1} r\right)\right]-\right. \\
\left.-i_{0} \alpha^{-1} \frac{\omega_{1}}{\omega_{2}\left(\beta_{3}^{2}-\beta_{2}^{2}\right)}\left[A_{22}(\alpha) J_{0}\left(\alpha \beta_{2} r\right)+B_{22}(\alpha) Y_{0}\left(\alpha \beta_{2} r\right)\right]\right\} \exp \left(i_{0} \alpha z\right) d \alpha \\
= \\
\psi=-\frac{1}{2 \pi} \int_{-\infty}^{\infty}\left\{\frac{\alpha^{-1} \beta_{2}}{\omega_{2}\left(\beta_{3}^{2}-\beta_{2}^{2}\right)}\left[A_{22}(\alpha) J_{1}\left(\alpha \beta_{2} r\right)+B_{22}(\alpha) Y_{1}\left(\alpha \beta_{2} r\right)\right]-\right. \\
\left.-\frac{2 i_{0} \alpha^{-1} v}{\omega_{2}\left(\beta_{3}^{2}-\beta_{1}^{2}\right)}\left[A_{11}(\alpha) J_{1}\left(\alpha \beta_{1} r\right)+B_{11}(\alpha) Y_{1}\left(\alpha \beta_{1} r\right)\right]\right\} \exp \left(i_{0} \alpha z\right) d \alpha .
\end{gathered}
$$

As a result of analysis according to the functions $\varphi$ and $\psi$ described by equations (11) they take the form: 


$$
\begin{gathered}
\varphi=\frac{1}{2 \pi} \int_{-\infty}^{\infty}\left\{\frac{\alpha^{-1} \beta_{1}}{\beta_{3}^{2}-\beta_{1}^{2}}\left[A_{11}(\alpha) J_{0}\left(\alpha \beta_{1} r\right)+B_{11}(\alpha) Y_{0}\left(\alpha \beta_{1} r\right)\right]+\right. \\
+\frac{i_{0} \alpha^{-1} \omega_{1}}{\omega_{2}\left(\beta_{3}^{2}-\beta_{2}^{2}\right)}\left[A_{22}(\alpha) J_{0}\left(\alpha \beta_{2} r\right)+B_{22}(\alpha) Y_{0}\left(\alpha \beta_{2} r\right)\right]+ \\
\left.+A_{12}(\alpha) J_{0}\left(\alpha \beta_{3} r\right)+B_{12}(\alpha) Y_{0}\left(\alpha \beta_{3} r\right)\right\} \exp \left(i_{0} \alpha z\right) d \alpha ; \\
\psi=-\frac{1}{2 \pi} \int_{-\infty}^{\infty}\left\{\frac{\alpha^{-1} \beta_{2}}{\omega_{2}\left(\beta_{3}^{2}-\beta_{2}^{2}\right)}\left[A_{22}(\alpha) J_{1}\left(\alpha \beta_{2} r\right)+B_{22}(\alpha) Y_{1}\left(\alpha \beta_{2} r\right)\right]-\right. \\
-\frac{2 i_{0} \alpha^{-1} v}{\omega_{2}\left(\beta_{3}^{2}-\beta_{1}^{2}\right)}\left[A_{11}(\alpha) J_{1}\left(\alpha \beta_{1} r\right)+B_{11}(\alpha) Y_{1}\left(\alpha \beta_{1} r\right)\right]- \\
\left.-\left[A_{21}(\alpha) J_{1}\left(\alpha \beta_{3} r\right)+B_{21}(\alpha) Y_{1}\left(\alpha \beta_{3} r\right)\right]\right\} \exp \left(i_{0} \alpha z\right) d \alpha
\end{gathered}
$$

Taking into account the peculiarities of the behavior of the Bessel functions of the first and second kind, the final expressions for the desired functions of displacements $U_{r}$ and $U_{z}$ write them on the basis of formulas (5), (7) and present in this form:

$$
\begin{aligned}
& U_{r}=-\frac{1}{2 \pi} \operatorname{Re} \int_{-\infty}^{\infty}\left[\omega_{3} A_{11}(\alpha) J_{1}\left(\alpha \beta_{1} r\right)+i_{0} \omega_{4} A_{22}(\alpha) J_{1}\left(\alpha \beta_{2} r\right)+\right. \\
& \left.+\alpha \beta_{3} A_{12}(\alpha) J_{1}\left(\alpha \beta_{3} r\right)-i_{0} \alpha \frac{b-\lambda c}{1-\lambda b} A_{21}(\alpha) J_{1}\left(\alpha \beta_{3} r\right)\right] \exp \left(i_{0} \alpha z\right) d \alpha ; \\
& \omega_{3}=\left[\beta_{1}^{2} \omega_{2}+2 v \frac{b-\lambda c}{1-\lambda b}\right] \omega_{2}^{-1}\left(\beta_{3}^{2}-\beta_{1}^{2}\right)^{-1} \\
& \omega_{4}=\beta_{2}\left[\omega_{1}+\frac{b-\lambda c}{1-\lambda b}\right] \omega_{2}^{-1}\left(\beta_{3}^{2}-\beta_{2}^{2}\right)^{-1} \\
& U_{z}=\frac{1}{2 \pi} \operatorname{Re} \int_{-\infty}^{\infty}\left[i_{0} \omega_{5} A_{11}(\alpha) J_{0}\left(\alpha \beta_{1} r\right)-\omega_{6} A_{22}(\alpha) J_{0}\left(\alpha \beta_{2} r\right)+\right. \\
& \left.+\alpha \beta_{3} \omega_{7} A_{21}(\alpha) J_{0}\left(\alpha \beta_{3} r\right)+i_{0} \alpha A_{12}(\alpha) J_{0}\left(\alpha \beta_{3} r\right)\right] \exp \left(-i_{0} \alpha z\right) d \alpha ; \\
& \omega_{7}=\omega_{2}(1-2 v)^{-1} ; \quad \omega_{5}=\beta_{1}\left(2 v \omega_{7}+\omega_{2}\right) \omega_{2}^{-1}\left(\beta_{3}^{2}-\beta_{1}^{2}\right)^{-1} ; \\
& \omega_{6}=\left(\omega_{1}-\frac{b-\lambda c}{1-\lambda b} \beta_{2}^{2} \omega_{2}\right)\left[\omega_{2}\left(\beta_{3}^{2}-\beta_{2}^{2}\right)\right]^{-1} .
\end{aligned}
$$

Finally, by separation the real and imaginary parts in formulas (2), the final expressions for the components of the displacement vector are: 


$$
\begin{aligned}
& U_{r}=-\frac{1}{\pi} \int_{0}^{\infty}\left\{\left[A_{1}(\alpha) U_{r}^{(1)}+A_{2}(\alpha) U_{r}^{(2)}+A_{3}(\alpha) U_{r}^{(3)}-A_{4}(\alpha) U_{r}^{(4)}\right] \cos \alpha z-\right. \\
& \left.-\left[B_{1}(\alpha) U_{r}^{(1)}-B_{2}(\alpha) U_{r}^{(2)}+B_{3}(\alpha) U_{r}^{(3)}+B_{4}(\alpha) U_{r}^{(4)}\right] \sin \alpha z\right\} d \alpha \\
& U_{r}=-\frac{1}{\pi} \int_{0}^{\infty}\left\{\left[A_{1}(\alpha) U_{r}^{(1)}-A_{2}(\alpha) U_{r}^{(2)}+A_{3}(\alpha) U_{r}^{(3)}-A_{4}(\alpha) U_{r}^{(4)}\right] \sin \alpha z+\right. \\
& \left.+\left[B_{1}(\alpha) U_{r}^{(1)}+B_{2}(\alpha) U_{r}^{(2)}+B_{3}(\alpha) U_{r}^{(3)}+B_{4}(\alpha) U_{r}^{(4)}\right] \cos \alpha z\right\} d \alpha ;
\end{aligned}
$$

where $A_{n}(\alpha)$ and $B_{n}(\alpha),(n=1,2,3,4)$ - arbitrary argument functions $\alpha$.

$$
\begin{aligned}
& U_{r}^{(1)}=\omega_{3} J_{1}\left(\alpha \beta_{1} r\right) ; U_{r}^{(2)}=\omega_{4} J_{1}\left(\alpha \beta_{2} r\right) ; \quad U_{r}^{(3)}=\alpha \beta_{3} J_{1}\left(\alpha \beta_{3} r\right) ; \\
& U_{r}^{(4)}=\alpha(b-\lambda c)(1-\lambda b)^{-1} J_{1}\left(\alpha \beta_{3} r\right) ; U_{z}^{(1)}=\omega_{5} J_{0}\left(\alpha \beta_{1} r\right) ; \\
& U_{z}^{(2)}=\omega_{6} J_{0}\left(\alpha \beta_{2} r\right) ; U_{z}^{(3)}=\alpha J_{0}\left(\alpha \beta_{3} r\right) ; \\
& U_{z}^{(4)}=\alpha \beta_{3} \omega_{7} J_{0}\left(\alpha \beta_{3} r\right) ; \omega_{7}=\omega_{2}(1-2 v)^{-1}
\end{aligned}
$$

Analytical dependences for the stress tensor components in the cylindrical coordinate system in ratios of Hooke's law [10]:

$$
\begin{aligned}
& \sigma_{r r}=\frac{q_{0}}{\pi} \int_{0}^{\infty}\left\{\left[A_{1}(\alpha) \sigma_{r r}^{(1)}+A_{2}(\alpha) \sigma_{r r}^{(2)}+\alpha A_{3}(\alpha) \sigma_{r r}^{(3)}-\alpha A_{4}(\alpha) \sigma_{r r}^{(4)}\right] \cos \alpha z-\right. \\
& \left.-\left[B_{1}(\alpha) \sigma_{r r}^{(1)}-B_{2}(\alpha) \sigma_{r r}^{(2)}+\alpha B_{3}(\alpha) \sigma_{r r}^{(3)}+\alpha B_{4}(\alpha) \sigma_{r r}^{(4)}\right] \sin \alpha z\right\} d \alpha \text {; } \\
& \sigma_{r r}^{(1)}=\frac{\omega_{3}}{r} \frac{1-2 v}{1-v} J_{1}\left(\alpha \beta_{1} r\right)-\alpha\left(\beta_{1} \omega_{3}-\frac{v}{1-v} \omega_{5}\right) J_{0}\left(\alpha \beta_{1} r\right) ; \\
& \sigma_{r r}^{(2)}=\frac{\omega_{4}}{r} \frac{1-2 v}{1-v} J_{1}\left(\alpha \beta_{2} r\right)-\alpha\left(\beta_{2} \omega_{4}+\frac{v}{1-v} \omega_{6}\right) J_{0}\left(\alpha \beta_{2} r\right) ; \\
& \sigma_{r r}^{(3)}=\frac{\beta_{3}}{r} \frac{1-2 v}{1-v} J_{1}\left(\alpha \beta_{3} r\right)-\alpha\left(\beta_{3}^{2}-\frac{v}{1-v}\right) J_{0}\left(\alpha \beta_{3} r\right) ; \\
& \sigma_{r r}^{(4)}=\frac{1}{r} \frac{1-2 v}{1-v} \frac{b-\lambda c}{1-\lambda b} J_{1}\left(\alpha \beta_{3} r\right)+\alpha \beta_{3}\left(\omega_{7}-\frac{b-\lambda c}{1-\lambda b} J_{0}\left(\alpha \beta_{3} r\right)\right. \\
& \sigma_{\theta \theta}=-\frac{q_{0}}{\pi} \int_{0}^{\infty}\left\{\left[A_{1}(\alpha) \sigma_{\theta \theta}^{(1)}+A_{2}(\alpha) \sigma_{\theta \theta}^{(2)}+\alpha A_{3}(\alpha) \sigma_{\theta \theta}^{(3)}+\alpha A_{4}(\alpha) \sigma_{\theta \theta}^{(4)}\right] \cos \alpha z-\right. \\
& \left.-\left[B_{1}(\alpha) \sigma_{\theta \theta}^{(1)}-B_{2}(\alpha) \sigma_{\theta \theta}^{(2)}-\alpha B_{3}(\alpha) \sigma_{\theta \theta}^{(3)}+\alpha B_{4}(\alpha) \sigma_{\theta \theta}^{(4)}\right] \sin \alpha z\right\} d \alpha
\end{aligned}
$$




$$
\begin{aligned}
& \sigma_{\theta \theta}^{(1)}=\frac{\omega_{3}}{r} \frac{1}{1-v} J_{1}\left(\alpha \beta_{1} r\right)+\alpha\left(\beta_{1} \omega_{3}-\omega_{5}\right) \frac{v}{1-v} J_{0}\left(\alpha \beta_{1} r\right) ; \\
& \sigma_{\theta \theta}^{(2)}=\frac{\omega_{4}}{r} \frac{1}{1-v} J_{1}\left(\alpha \beta_{2} r\right)+\alpha\left(\beta_{2} \omega_{4}+\omega_{6}\right) \frac{v}{1-v} J_{0}\left(\alpha \beta_{2} r\right) ; \\
& \sigma_{\theta \theta}^{(3)}=\frac{\beta_{3}}{r} \frac{1}{1-v} J_{1}\left(\alpha \beta_{3} r\right)+\frac{\alpha \nu}{1-v}\left(\beta_{3}^{2}-1\right) J_{0}\left(\alpha \beta_{3} r\right) \text {; } \\
& \sigma_{\theta \theta}^{(4)}=\frac{1}{r} \frac{b-\lambda c}{1-\lambda b} \frac{1}{1-v} J_{1}\left(\alpha \beta_{3} r\right)-\alpha \beta_{3} \frac{v}{v-1}\left(\omega_{7}-\frac{b-\lambda c}{1-\lambda b} J_{0}\left(\alpha \beta_{3} r\right)\right. \text {; } \\
& \sigma_{z z}=-\frac{q_{0}}{\pi} \int_{0}^{\infty}\left\{\left[A_{1}(\alpha) \sigma_{z z}^{(1)}+A_{2}(\alpha) \sigma_{z z}^{(2)}+A_{3}(\alpha) \sigma_{z z}^{(3)}+A_{4}(\alpha) \sigma_{z z}^{(4)}\right] \cos \alpha z-\right. \\
& \left.-\left[B_{1}(\alpha) \sigma_{z z}^{(1)}-B_{2}(\alpha) \sigma_{z z}^{(2)}+B_{3}(\alpha) \sigma_{z z}^{(3)}+B_{4}(\alpha) \sigma_{z z}^{(4)}\right] \sin \alpha z\right\} d \alpha \\
& \sigma_{z z}^{(1)}=\left(\frac{v}{1-v} \beta_{1} \omega_{3}-\omega_{5}\right) J_{0}\left(\alpha \beta_{1} r\right) ; \quad \sigma_{z z}^{(2)}=\left(\frac{1}{1-v} \beta_{2} \omega_{4}+\omega_{6}\right) J_{0}\left(\alpha \beta_{2} r\right) ; \\
& \sigma_{z z}^{(3)}=\alpha\left(\frac{1}{1-v} \beta_{3}^{2}-1\right) J_{0}\left(\alpha \beta_{3} r\right) ; \sigma_{\theta \theta}^{(4)}=\alpha \beta_{3}\left(\omega_{7}-\frac{v}{1-v} \frac{b-\lambda c}{1-\lambda b}\right) J_{0}\left(\alpha \beta_{3} r\right) ; \\
& \sigma_{r z}=\frac{q_{1}}{\pi} \int_{0}^{\infty}\left\{\left[A_{1}(\alpha) \sigma_{r z}^{(1)}+A_{2}(\alpha) \sigma_{r z}^{(2)}-A_{4}(\alpha) \sigma_{r z}^{(4)}\right] \sin \alpha z+\right. \\
& \left.+\left[B_{1}(\alpha) \sigma_{r z}^{(1)}-B_{2}(\alpha) \sigma_{r z}^{(2)}+B_{2}(\alpha) \sigma_{r z}^{(4)}\right] \cos \alpha z\right\} d \alpha \\
& \sigma_{r z}^{(1)}=\left(\omega_{3}-\beta_{1} \omega_{5}\right) J_{1}\left(\alpha \beta_{1} r\right) ; \quad \sigma_{r z}^{(2)}=\left(\omega_{4}+\beta_{2} \omega_{6}\right) J_{1}\left(\alpha \beta_{2} r\right) ; \\
& \sigma_{r z}^{(4)}=\alpha\left[\frac{b-\lambda c}{1-\lambda b}-\beta_{3}^{2} \omega_{7}\right] J_{1}\left(\alpha \beta_{3} r\right) .
\end{aligned}
$$

\section{Analysis of the results}

Thus, the obtained dependences for the components of the displacement vector (14) and the stress tensor have sufficient functional arbitrariness to meet the boundary conditions (3) and (4).

It should be noted that the presence of arbitrary constants $\lambda$ and $B$ in formulas (15) and (16) provides sufficient functional arbitrariness to meet the boundary conditions (5) and (3). The values of the constants $\lambda$ and $b$ are determined numerically on a computer.

In order to establish the influence of natural and possible stresses arising during mine excavation, on the indicators of the resistance of rocks to mechanical destruction, one should determine from the boundary conditions (3) and (4) the functions $A_{n}(\alpha)$ and $B_{n}(\alpha)$.

Using the found form of the defining equalities (15) and (16), we satisfy the boundary conditions on the lateral surface and the end of the cylindrical body. Substituting the appropriate expressions of the stress tensor components in the conditions (3) and (4), we 
obtain the following two systems of integral equations the relatively sought functions $A_{n}(\alpha)$ and $B_{n}(\alpha),(n=1,2,3,4)$, where:

$$
\begin{aligned}
& A_{1}(\alpha)=F_{2}(\alpha) \nabla_{27}-\alpha A_{3}(\alpha) \nabla_{14} / \nabla_{11} \nabla ; \\
& A_{2}(\alpha)=F_{2}(\alpha) \nabla_{28}-\alpha A_{3}(\alpha) \nabla_{17} / \nabla_{11} \nabla ; \\
& A_{3}(\alpha)=\beta_{3} \nabla_{11} \nabla\left(\alpha^{2} \nabla_{26}\right)^{-1} \int_{0}^{1} \chi(\xi) \cos \left(\alpha \beta_{3} \xi\right) d \xi ; \\
& A_{4}(\alpha)=-\alpha A_{3}(\alpha) \nabla_{22} / \nabla_{11} \nabla ; \\
& B_{1}(\alpha)=\frac{\pi}{q_{1}} F_{1}(\alpha) \Delta_{27}+\alpha B_{3}(\alpha) \Delta_{18} ; \\
& B_{2}(\alpha)=\frac{\pi}{q_{1}} F_{1}(\alpha) \Delta_{28}+\alpha B_{3}(\alpha) \Delta_{22} ; \\
& B_{3}(\alpha)=\left(\alpha^{2} \tau_{r z}^{(4)} \Delta_{25}\right)^{-1} \int_{0}^{1} \chi_{1}(\xi) \sin \left(\alpha \beta_{3} \xi\right) d \xi ; \\
& B_{4}(\alpha)=\alpha B_{3}(\alpha) \Delta_{25} .
\end{aligned}
$$

The calculation of the stress tensor parameters is carried out by numerical integration improper integrals in formuls (16), based on the program for the computer.

\section{References}

1. Baron, L.I., Glatman, L.B. (1966). Kontaktnaya prochnost gornyh porod. Moskva: Nedra

2. Baron, L.I., Azerskaya, K.F. (1977). Kontaktnaya prochnost kak kriterij soprotivlyaemosti gornyh porod razrusheniyu tangencialnymi diskovymi sharoshkami, Gornye mashiny i avtomatika, 8 (137), 11-12

3. Perepelica, V.G., Kolomiec, A.N., Shmatovskij, L.D. (2012) Osobennosti napryazhennogo sostoyaniya gornogo massiva $v$ processe provedeniya vyrabotki, Dopovidi NAN Ukrayiny, (5), 57-61

4. Bulat, A.F., Shmatovskij, L.D., Kolomiec, A. N. (2012). Vliyanie formy zaboya na napryazhennoe sostoyanie massiva vblizi gornoj vyrabotki, Varshava: Teorety chni osnovy budivny 'cztva, (20), 171-178

5. Protasov, Yu.I. (1985). Teoreticheskie osnovy mekhanicheskogo razrusheniya gornyh porod. Moskva: Nedra

6. Ehjgeles, R.M. (1971). Razrushenie gornyh porod pri burenii. Moskva: Nedra

7. Lyav, A. (1935). Matematicheskaya teoriya uprugosti. Moskva. ONTI NKTI SSSR

8. Sneddon, I. (1955). Preobrazovaniya Furye, Moskva: Izdatelstvo inostrannoy literatury

9. Vatson, G.N. (1949). Teoriya Besselevyh funkcij. Moskva: Izdatelstvo inostrannoy literatury

10. Ehdelshtejn, E.I., Korolko, V.I., Ehjgeles, R.M., Ehstrin Yu.Ya. (1966). Teoreticheskie i ehksperimental'nye issledovaniya mekhanizma razrusheniya gornyh porod, Alma-Ata: Problemy mekhaniki gornyh porod: Materialy Vsesoyuznoy nauchnoy konferencii, 473483 